\title{
Programa de Articulación de las funciones de Docencia, Investigación, Extensión y Proyección Social en la Facultad de Administración y Negocios
}

\author{
Ana Beatriz Blanco-Ariza \\ Gisella Rossy Rodríguez-Calderón \\ Prince Luz Torres-Salazar \\ Karelis Barrios Hernández \\ Diana Escamilla Narváez \\ Sandra Susana Jaimes Mora
}

Durante el proceso formativo, los estudiantes de la Facultad de Administración y Negocios de la Universidad Simón Bolívar tendrán la oportunidad de desarrollar de manera transversal a lo largo de los diferentes programas académicos, una serie de ejercicios estratégicos de articulación, que responderán a objetivos de las asignaturas de su formación profesional y potenciarán el pensamiento investigativo, crítico, sistemático y contextualizado, que complementará su formación integral porque estará en contacto permanente con la teoría y la práctica.

Adicional a esto, desde los grupos de investigación adscritos a la facultad, a saber: Gestión Organizacional, Pensamiento Contable y Gestión Internacional, Grupo Innovación y Desarrollo Empresarial y, Grupo Gestión de la Innovación y Emprendimiento, se diseñarán e implementarán una serie de talleres formativos a lo largo de cada semestre, lo que permitirá nivelar las competencias de los docentes y estudiantes que tengan deficiencias en aspectos de aplicación de normas de la Asociación Americana de Psicología 
(APA, por sus siglas en inglés), redacción, metodología de la investigación y manejo de las guías establecidas para la realización de los ejercicios.

Este programa articulador de las funciones de docencia, investigación y, extensión y proyección social se materializará en el aula de clases a través de los profesores del área profesional de cada programa académico de la facultad, dado que permitirá una mejor articulación de estas funciones; además de los avances y desarrollos investigativos que se pretendan lograr bajo esta nueva estrategia, cabe resaltar que dentro del proceso formativo, las otras asignaturas servirán de apoyo para el acompañamiento de estudiantes y profesores.

Es importante conocer que, para el desarrollo de la estrategia, se contempla la escogencia de las asignaturas del área profesional (una asignatura por cada semestre), en ese sentido, en reuniones realizadas con la coordinación de docencia de la facultad, se acordó la siguiente línea de asignaturas en cada programa académico (ver Tabla 3).

Tabla 3. Asignaturas articuladas a la estrategia PADIE por programa de facultad

\begin{tabular}{ll}
\hline & \multicolumn{1}{c}{ PROGRAMA DE ADMINISTRACIÓN DE EMPRESAS } \\
\hline SEMESTRE & ASIGNATURAS \\
I & Fundamentos de Administración y Gerencia \\
II & Teorías y Enfoques Contemporáneos de la Administración - Metodología de la Investigación Cualitativa \\
III & Procesos Administrativos - Metodología de la Investigación Cuantitativa \\
IV & Electiva Básica Profesional \\
V & Desarrollo y Comportamiento Organizacional \\
VI & Marketing \\
VII & Gestión del Talento Humano I, Investigación de Mercados \\
VIII & Electiva Profesional \\
IX & Gerencia Estratégica, Gerencia de Servicio, Electiva Profesional, Gestión de Pymes \\
X & Práctica Empresarial \\
\hline
\end{tabular}




\begin{tabular}{ll}
\hline & \multicolumn{1}{c}{ PROGRAMA DE CONTADURÍA PÚBLICA } \\
\hline SEMESTRE & ASIGNATURAS \\
I & Contabilidad I \\
II & Contabilidad II \\
III & Métodos y Metodología Científica I \\
IV & Métodos y Metodología Científica II - Teoría Contable \\
V & Comercio Internacional \\
VI & Investigación I \\
VII & Investigación II \\
VIII & Laboratorio Contable \\
IX & Formulación y Evaluación de Proyectos de Inversión \\
\hline
\end{tabular}

PROGRAMA DE COMERCIO Y NEGOCIOS INTERNACIONALES

\begin{tabular}{ll}
\hline SEMESTRE & ASIGNATURAS \\
I & Comercio Exterior I, Introducción a la Administración \\
II & Comercio Exterior II, Fundamentos de Negocios Internacionales \\
III & Metodología de la Investigación I, Desarrollo, Cultura y Globalización \\
IV & Metodología de la Investigación II, Globalización y Competitividad \\
V & Investigación Formativa, Mercadeo Básico Internacional \\
VI & Investigación Formativa II, Emprendimiento Empresarial \\
VII & Investigación Formativa III, Formulación y Evaluación de Proyectos \\
VIII & Administración Portuaria y de Transporte, Diplomacia y Relaciones Internacionales \\
IX & Plan Exportador \\
\hline & PROGRAMA TÉCNICO-PROFESIONAL EN PROCESOS PUBLICITARIOS Y DE MERCADEO \\
\hline SEMESTRE & ASIGNATURAS \\
I & Mercadeo \\
II & Publicidad \\
III & Seminario de Investigación \\
IV & Investigación de Mercados \\
\hline & PROGRAMA TÉCNICO-PROFESIONAL EN OPERACIONES PORTUARIAS \\
\hline SEMESTRE & ASIGNATURAS \\
I & Unitarización de la Carga, Tarja de la Carga \\
II & Empaque y Embalaje, Seguridad de la Carga \\
III & Seminario de Investigación, Soluciones Ambientales Portuarias \\
IV & Administración del Talento Humano, Trámite de Operaciones Aduaneras \\
\hline & Fuente: Elaboración propia a partir de los planes de estudio de los programas \\
&
\end{tabular}




\section{FASES DE EJERCICIOS DE ARTICULACIÓN}

Dado que en la Facultad de Administración y Negocios se ha implementado esta estrategia de primero a décimo semestre, se han estructurado dos fases que han permitido determinar el grado de complejidad de cada uno de los ejercicios que realizarán los estudiantes semestre a semestre. Las fases se han definido de la siguiente manera en la Tabla 4.

Tabla 4. Relación de fases de los ejercicios de articulación

\begin{tabular}{lc}
\hline \multicolumn{1}{c}{ FASES } & SEMESTRE \\
\hline BÁSICA & 1 a 4 \\
PROFESIONAL & 5 a 10 \\
\hline \multicolumn{2}{c}{ Fuente: Elaboración propia }
\end{tabular}

\section{Fase básica}

Compuesta de primero a cuarto semestre, se le denomina fase básica, porque durante este tiempo el estudiante estará en proceso de formación de competencias generales, apoyado en las asignaturas del área básica como la investigativa desde: Metodología de la Investigación Cualitativa y Cuantitativa, además de las asignaturas del área profesional y de los talleres de formación que se dictarán como alternativas para promover una relación más viva entre la teoría y la práctica de profesores y estudiantes.

Bajo esta premisa los estudiantes realizan ejercicios de articulación básicos como consultar diferentes bases de datos para construir un estado de arte sobre un tema específico, visitas empresariales, asistencia a eventos, entre otros. El propósito de este nivel es que estudiantes y profesores den cuenta de la epistemología de la profesión y que inicien el relacionamiento vivencial con el propósito de enseñarles a construir conocimiento a partir de los saberes y el hacer. 


\section{Fase profesional}

Este nivel abarca de quinto a décimo semestre. Se llama profesional porque en él, los estudiantes ya han adquirido la formación básica que necesita el futuro administrador de empresas y empieza el desarrollo de asignaturas propias de la disciplina, lo cual requiere un nivel de exigencia mayor. Es por esto, que los ejercicios de relacionamiento se tornan enfocados en las competencias procedimentales o del saber hacer, enmarcados en la construcción de artículos, intervención en las empresas a través de problemáticas planteadas y definidas, participación como ponentes en eventos, trabajo con comunidades y empresas de diferentes sectores económicos, participación en formación con expertos temáticos, construcción de estudios de casos, desarrollo de proyectos, entre otras actividades.

\section{EJERCICIOS DE ARTICULACIÓN}

Los profesores podrán escoger dentro de una gama de opciones, las que más se ajusten a las temáticas y al desarrollo de la actividad propuesta dentro del aula de clase. Es así, como se ha establecido una serie de ejercicios por semestre todo de acuerdo con las características propias de cada programa académico de la facultad. En una construcción colectiva se determinan las diferentes alternativas en términos de ejercicios que podrán realizar los estudiantes; la fase básica será de preparación, esto les permitirá la construcción de las bases que deberán aplicar en la siguiente instancia, la fase profesional. En esta segunda fase es donde se evidenciará realmente la verdadera articulación de las funciones de docencia, investigación, extensión y proyección social.

Los profesores de las diferentes asignaturas asesorarán a los es- 
tudiantes, lo mismo que los docentes-investigadores de los grupos que tiene el programa, esto como mecanismo de intervención para el fortalecimiento de la cultura investigativa al interior de la Facultad de Administración y Negocios. Otro de los objetivos que se pretende con esta estrategia es identificar en los estudiantes habilidades para la investigación y motivarlos a participar como auxiliares en los grupos y a vincularse a los programas institucionales de formación investigativa de la institución como el Programa Institucional de Semilleros de Investigación e Innovación $\left(\mathrm{SI}^{2}\right)$.

Este Programa de Articulación de las funciones de Docencia, Investigación, Extensión y Proyección Social se convierte en un punto articulador entre la facultad y el programa institucional de Jóvenes Investigadores e Innovadores de la Universidad Simón Bolívar, siendo esta una estrategia institucional para la formación y fomento de la cultura investigativa, desde el espacio de cada programa académico.

Esta estrategia institucional es coordinada por el Departamento de Formación para la Investigación (DEFI), a través de los responsables de investigación de cada facultad, así como las coordinaciones de los centros de investigación. Lo que se pretende a su vez es que las actividades que realicen los estudiantes del semillero se enmarquen entre los ejercicios del PADIE. A continuación la Tabla 5 presenta el conglomerado de ejercicios de articulación que los profesores y estudiantes pueden apropiar según la dimensión y disciplina de la asignatura que vincule a la estrategia del programa. 
Tabla 5. Ejercicios de articulación de las funciones docencia, investigación y extensión

\begin{tabular}{|c|c|c|}
\hline SEM & EJERCICIO & PRODUCTO \\
\hline 1 & Línea de tiempo & Posters, infografía \\
\hline \multirow[b]{2}{*}{2} & Revisión bibliográfica & Monografía \\
\hline & $\begin{array}{l}\text { Diseño y aplicación de instrumentos } \\
\text { cualitativos }\end{array}$ & Informe de sistematización de la actividad \\
\hline \multirow{3}{*}{3} & $\begin{array}{l}\text { Diseño y aplicación de instrumentos } \\
\text { cualitativos }\end{array}$ & Informe de sistematización de la actividad \\
\hline & $\begin{array}{l}\text { Diseño y aplicación de instrumentos } \\
\text { cuantitativos }\end{array}$ & $\begin{array}{l}\text { Informe de sistematización y análisis de } \\
\text { resultados }\end{array}$ \\
\hline & Revisión bibliográfica & Ensayo \\
\hline \multirow{4}{*}{4} & $\begin{array}{l}\text { Diseño y aplicación de instrumentos } \\
\text { cualitativos }\end{array}$ & Informe de sistematización de la actividad \\
\hline & $\begin{array}{l}\text { Diseño y aplicación de instrumentos } \\
\text { cuantitativos }\end{array}$ & $\begin{array}{l}\text { Informe de sistematización y análisis de } \\
\text { resultados }\end{array}$ \\
\hline & Revisión de estado del arte & Propuesta de investigación, ensayo \\
\hline & Organización de eventos & Informe de actividad \\
\hline \multirow{5}{*}{5} & $\begin{array}{l}\text { Organización y participación en Work- } \\
\text { shop internos }\end{array}$ & Informe de Workshop \\
\hline & $\begin{array}{l}\text { Análisis global de tendencias en rela- } \\
\text { ción con los productos identificados } \\
\text { para internacionalización en la región. }\end{array}$ & Matriz de Inteligencia de Mercados \\
\hline & $\begin{array}{l}\text { Participación como ponentes en } \\
\text { eventos }\end{array}$ & Ponencia publicada \\
\hline & $\begin{array}{l}\text { Formulación de problemas de inves- } \\
\text { tigación, formulación de objetivos y } \\
\text { revisión bibliográfica }\end{array}$ & Formulación de anteproyecto de investigación \\
\hline & Diseño y validación de instrumentos & Instrumento validado \\
\hline \multirow{5}{*}{6} & $\begin{array}{l}\text { Formulación de problemas de investi- } \\
\text { gación y objetivos, revisión bibliográfi- } \\
\text { ca y diseño metodológico }\end{array}$ & Anteproyecto de investigación \\
\hline & $\begin{array}{l}\text { Identificación de las capacidades } \\
\text { regionales en producción y exporta- } \\
\text { ción de productos de acuerdo con las } \\
\text { demandas identificadas en la matriz de } \\
\text { inteligencia de mercados. }\end{array}$ & $\begin{array}{l}\text { Informe de análisis y diagnóstico de } \\
\text { capacidades }\end{array}$ \\
\hline & Análisis y comparación de casos & Informe de casos \\
\hline & $\begin{array}{l}\text { Aplicación de instrumento de investi- } \\
\text { gación cualitativa }\end{array}$ & $\begin{array}{l}\text { Informe de sistematización y análisis de } \\
\text { resultados }\end{array}$ \\
\hline & $\begin{array}{l}\text { Creación de un producto o servicio } \\
\text { innovador }\end{array}$ & Plan de marketing \\
\hline
\end{tabular}


Programa de Articulación de las Funciones de Docencia, Investigación, Extensión y Proyección Social (PADIE). Un enfoque desde la Facultad de Administración y Negocios

\begin{tabular}{|c|c|c|}
\hline SEM & EJERCICIO & PRODUCTO \\
\hline \multirow{4}{*}{7} & Construcción de marco teórico & Artículo de revisión \\
\hline & Realización de visita empresarial & Informe de visita \\
\hline & $\begin{array}{l}\text { Diseño y aplicación de instrumentos } \\
\text { cualitativos }\end{array}$ & $\begin{array}{l}\text { Informe de sistematización y análisis de } \\
\text { resultados }\end{array}$ \\
\hline & $\begin{array}{l}\text { Diseño y aplicación de instrumentos } \\
\text { cuantitativos }\end{array}$ & $\begin{array}{l}\text { Informe de sistematización y análisis de } \\
\text { resultados }\end{array}$ \\
\hline \multirow{6}{*}{8} & $\begin{array}{l}\text { Construcción y análisis de estudio de } \\
\text { casos }\end{array}$ & Casos publicados \\
\hline & $\begin{array}{l}\text { Identificación de posible mercado } \\
\text { objetivo y análisis de variables } \\
\text { macroeconómicas }\end{array}$ & Informe de estudio de mercado internacional \\
\hline & Realización de visita empresarial & Informe de visita \\
\hline & $\begin{array}{l}\text { Organización y participación en Work- } \\
\text { shop externo }\end{array}$ & Informe de actividad \\
\hline & $\begin{array}{l}\text { Preparación de foros de divulgación } \\
\text { parcial de los resultados con sector } \\
\text { externo }\end{array}$ & Informe de actividad \\
\hline & Organización y participación en MOEA & Informe de actividad \\
\hline \multirow{3}{*}{9} & $\begin{array}{l}\text { Realización de direccionamiento estra- } \\
\text { tégico a una Mipymes }\end{array}$ & Plan estratégico \\
\hline & $\begin{array}{l}\text { Diagnóstico y estudio de alterna- } \\
\text { tivas de solución a problemáticas } \\
\text { empresariales }\end{array}$ & Plan de mejoramiento \\
\hline & $\begin{array}{l}\text { Formulación de plan estratégico de } \\
\text { internacionalización }\end{array}$ & Plan exportador \\
\hline 10 & $\begin{array}{l}\text { Diagnóstico y estudio de alterna- } \\
\text { tivas de solución a problemáticas } \\
\text { empresariales }\end{array}$ & Plan de mejoramiento \\
\hline
\end{tabular}

Fuente: Elaboración propia

Tanto el rol de los profesores de las asignaturas como el de los investigadores es potenciar el desarrollo de las competencias de los estudiantes, tomando como fundamento conocimientos específicos de la formación de cada programa académico de la facultad para ser socializados al final de cada semestre en un acto público de amplio conocimiento para la comunidad estudiantil.

Cabe resaltar que, al finalizar el ejercicio de articulación, cada profesor escoge los tres mejores trabajos del aula de su clase y los remite a la Coordinación de Investigaciones de la facultad para 
su posterior evaluación y de allí seleccionar el mejor trabajo por semestre. Este trabajo seleccionado como el mejor se postulará para la revista estudiantil Liderazgo Estratégico, los dos trabajos restantes seleccionados entrarán a lista de espera en la revista para ser publicados en los siguientes números.

Para mayor entendimiento del proceso y de la forma como opera la estrategia se presenta la Figura 1, para que el lector contemple la representación gráfica del modelo propuesto que actualmente opera en los diferentes programas académicos de la facultad.

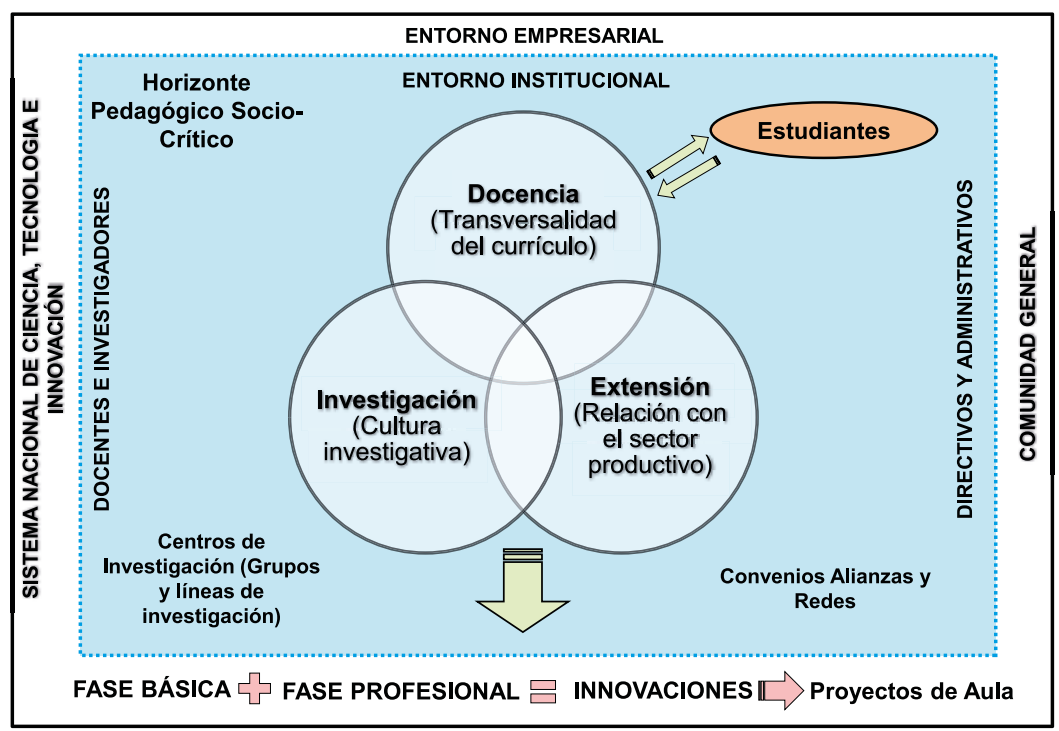

Figura 1. Modelo general de la estrategia PADIE Fuente: Elaboración propia

\section{RESPONSABILIDADES DE LOS ACTORES}

Es importante conocer que la implementación de la estrategia PADIE requiere una serie de consideraciones que se deben tener en cuenta para llegar a feliz término con el propósito que se pretende alcanzar: "Una comunidad académica fomentando la cultura investigativa y la articulación de las funciones de Docencia, Inves- 
tigación, Extensión y Proyección Social al interior de la facultad". Por esta razón las diferentes coordinaciones de funciones de la facultad tendrán las siguientes responsabilidades:

\section{Coordinación de Docencia}

- Coordinar con los directores de cada programa académico el listado de docentes y asignaturas que trabajarán la estrategia cada inicio de semestre

- Remitir a las Coordinaciones de Investigación, Extensión y Proyección Social el listado de docentes y asignaturas de cada programa académico

- Suministrar al docente los lineamientos necesarios para tal fin

- Hacer en conjunto con los directores de programa el seguimiento a las instrucciones que serán dadas desde las Coordinaciones de Investigación, Extensión y Proyección Social sobre las fechas de entrega y sustentaciones de trabajos, así como el seguimiento de la estrategia durante todo el semestre

- Revisar que la implementación de la estrategia está definida en cada una de las parcelaciones de los docentes

- Plantear las fechas de entrega de avances y entrega final de los ejercicios a realizar en cada semestre

- Organizar en conjunto con los directores de programas de la facultad, la premiación de los trabajos sobresalientes al final de cada semestre

- Hacer entrega a la Coordinación de Investigación al finalizar cada semestre, con su respectivo aval, de manera digital, los tres mejores trabajos seleccionados por cada profesor para someterlos a evaluación y postulación en la revista estudiantil de la facultad. 


\section{Coordinación de Investigación}

- Presentar a los profesores encargados de la estrategia las líneas, grupos de investigación y proyectos que se trabajan al interior de la facultad

- Programar los talleres sobre competencias investigativas de profesores y estudiantes

- Explicar a los profesores encargados de la estrategia, el propósito de cada uno de los ejercicios escogidos por la actividad en el aula

- Entregar todos los lineamientos para los esquemas de cada ejercicio escogido

- Asesorar a través de los diferentes grupos de investigación en temas relacionados con el área investigativa

- Enviar los mejores trabajos para postulación en las revistas de la facultad.

\section{Coordinación de Extensión y Proyección Social}

- Coordinar con las funciones de docencia e investigación los diferentes tipos de ejercicios que se realizarán en la fase profesional (quinto a noveno semestre)

- Establecer la relación con las empresas, gremios, comunidades o Estado que sean requeridas por el docente o la asignatura

- Programar las visitas empresariales por áreas de formación

- Invitar a actores empresariales y académicos para afianzar las competencias profesionales

- Programar reuniones con los profesores de quinto a décimo semestre para velar por la articulación de esta función en la fase profesional

- Medir el impacto que genera la articulación de esta estrategia a través de los diferentes ejercicios realizados por los estudiantes. 


\section{Profesores}

- Coordinar y concretar con las funciones docencia, investigación, extensión y proyección social, la propuesta de ejercicio de articulación que realizarán durante el semestre

- Escoger en cada salón de clase asignado los tres mejores trabajos y presentarlos ante la dirección de su programa académico

- Conocer las líneas de investigación de la facultad para enmarcar sus ejercicios en cada una de ellas y hacerlas visibles para problematizarlas

- Exigir las características solicitadas por la facultad para la presentación de los trabajos realizados

- Hacer uso de las herramientas de las Tecnologías de Información y Comunicación (TIC) como la Plataforma Moodle del programa para la comunicación y asesoría de estudiantes

- Incentivar el uso de las bases de datos disponibles la institución para la realización de los ejercicios de articulación

- Socializar con los estudiantes sobre la importancia de la propiedad intelectual en cada uno de los trabajos entregados

- Incluir dentro de su parcelación fechas y cronogramas de trabajos para la realización del ejercicio escogido

- Consultar con carácter obligatorio la producción investigativa generada por la facultad, con el propósito de utilizarla como material para el desarrollo de los ejercicios de articulación.

\section{Estudiantes}

- Hacer entrega del ejercicio derivado de la estrategia

- Cumplir con las fechas de entrega pactadas en la parcelación docente y con el profesor 
- Conocer las líneas de investigación de la facultad

- Cumplir con las características solicitadas por la facultad para la presentación de los trabajos

- Hacer uso de las normas APA en todos los ejercicios presentados

- No cometer plagio en los documentos presentados.

Para la correcta implementación de la estrategia se hace necesaria una construcción colectiva y compromiso por parte de los participantes anteriormente enunciados, de tal forma que se cuente con la debida plataforma de capacidades internas que permitan la generación de conocimientos aplicados útiles y pertinentes desde la misión investigativa de la facultad, y que aporte a la meta principal del programa PADIE, contribuir a la formación integral mediante la articulación de las dimensiones de docencia, investigación y extensión en el ejercicio aplicado en la formación de los profesionales de las Ciencias Administrativas, Económicas y Contables; dinamizando los saberes clásicos de la disciplina y aportándole al desarrollo nacional con procedimientos analíticos de rigor e impacto sobre el medio.

\footnotetext{
Cómo citar este capítulo:

Blanco-Ariza, A., Rodríguez-Calderón, G., Torres Salazar, P., Barrios Hernández, K., Escamilla Narváez, D. \& Jaimes Mora, S. (2017). Programa de Articulación de las funciones de Docencia, Investigación, Extensión y Proyección Social en la Facultad de Administración y Negocios. En: Blanco-Ariza, A. \& Rodríguez-Calderón, G. (coord.). Programa de Articulación de las Funciones de Docencia, Investigación, Extensión y Proyección Social (PADIE). Un enfoque desde la Facultad de Administración y Negocios. (pp.47-59). Barranquilla, Colombia: Ediciones Universidad Simón Bolívar.
} 